\title{
CÓMO INFLUYEN LA CONDUCTA DE LOS EMPLEADOS, LA SATISFACCIÓN DE LOS CLIENTES Y EL INTERÉS DE INVERTIR DEL ACCIONISTA EN LA EXPANSIÓN Y RENTABILIDAD DE LAS EMPRESAS COMERCIALES
}

\author{
Luis Márquez Guevara* \\ E-mail:Imarquezg@unmsm.edu.pe
}

\begin{abstract}
RESUMEN
Una de las formas de mejorar el crecimiento y la rentabilidad de una organización es tener una visión de la empresa como un lugar atractivo para trabajar; un lugar interesante para comprar, y un lugar interesante para invertir. Esta trilogía indica que si el comportamiento del trabajador con el cliente es correcto; si el cliente se siente satisfecho con el producto que se vende en esta empresa, porque cubre adecuadamente sus necesidades; y si mejora la rentabilidad de los accionistas, estamos ante una empresa en desarrollo y rentable.

Los resultados de esta trilogía se conocen a través de un Cuadro de Mando Integral por medio de un adecuado Control de Gestión, medidas correctivas que permitirán mantener en forma favorable la aplicación de la mencionada trilogía.
\end{abstract}

Palabras Clave: Conducta, satisfacción, interés, expansión y rentabilidad.

\section{ABSTRACT}

One way to improve the growth and profits of a corporate is having a vision of business as a pleasant place to work, an interesting place to buy and an interesting place to invest .This trilogy shows that if the worker behavior to the customer is correct, if the client gets satisfied with the product that the company sells, because it covers adequately his needs; and if enhance the profits of shareholders, we are facing an developed and profitable corporate.

The results of this trilogy are known through a Balanced Scorecard by a properly management control, this corrective measures that will permit to keep in favorable ways the application of this trilogy.

Key Words: Behavior, satisfaction, interest, expansion, profitability.

* Licenciado en Administración. Presidente de la ONG ECO. Profesor Asociado de la Facultad de Ciencias Administrativas (UNMSM). 


\section{INTRODUCCION}

Se observó y se analizó que muchas empresas comerciales no podían mejorar su crecimiento ni su rentabilidad debido a la conducta disfuncional de los empleados, así como también a la insatisfacción de los clientes con respecto a los productos que adquieren en menor volumen de ventas; que posteriormente afectaba a la rentabilidad de los activos, perjudicando a los accionistas de la empresa.

El presente estudio pretende establecer estrategias para que los empleados consideren a su centro laboral como un lugar atractivo para trabajar; para que los clientes consideren a la empresa como un lugar interesante para comprar, y finalmente para que los accionistas valoren a la organización como un lugar favorable para invertir.

La mayoría de los estudios similares encontrados son de carácter aislado e individual: cada estudio se ha referido sólo al empleado, o solo al cliente o sólo al accionista; casi nada hemos encontrado en cuanto al estudio de la trilogía empleado-cliente-accionista.

Además de esta trilogía, existen otros factores tales como los administradores, acreedores, la comunidad y otros que están relacionados con la responsabilidad social de la empresa, que no son objeto del presente estudio, pero que es importante tener en cuenta toda vez que el factor económico es indispensable para que la empresa comercial pueda cumplir con sus responsabilidades sociales. Si no logra utilidades no tendrá recursos para mejorar el producto y producirlo a menor costo para elevar las remuneraciones de los empleados y para atraer más capitales que permitan la expansión o incremento de volumen de ventas de la empresa comercial.
El presente estudio tiene como objetivo principal lograr una gran expansión e incrementar la rentabilidad de la inversión de las empresas comerciales.

\section{MÉTODOS}

El tipo de estudio es de investigación explicativa y de carácter cuantitativa por el uso estadístico-encuesta que se utilizó.

El problema se sistematizó de la siguiente manera: los factores conducta de los empleados, la imagen que tienen los clientes de la empresa, y el interés de invertir por parte de los accionistas, influyen en la expansión o volumen de ventas y en la rentabilidad de la inversión.

Se estableció como hipótesis: «La variación de los tres factores de conducta de los empleados, la imagen que tienen los clientes de la empresa y el interés de invertir por parte de los accionistas afectan en la variación de aumentar o disminuir la expansión y la rentabilidad de la empresa», teniendo en cuenta que "La aplicación de un solo factor, ya sea de los empleados, de los clientes o de los accionistas, no afecta la variación un aumento de la expansión y rentabilidad».

Estos dos conceptos esenciales de la hipótesis nos permitieron identificar las variables independientes, que son los factores empleados, clientes y accionistas; $y$ las variables dependientes, que son expansión y rentabilidad.

Los indicadores de las variables independientes y dependientes se obtuvieron de la siguiente manera (ver Cuadro 1):

- El diseño muestral se compone de cincuenta empresas de tipo comercial y ubicadas en Lima Metropolitana, cada una con un promedio de

Cuadro 1

Indicadores de las Variables Independientes y Dependientes

\begin{tabular}{|l|l|}
\hline \multicolumn{1}{|c|}{ VARIABLES INDEPENDIENTES } & \multicolumn{1}{c}{ INDICADORES } \\
\hline Conducta del Empleado & - Remuneraciones equitativas. \\
& - Consideración al personal en el trato. \\
& - Buenas condiciones ambientales de trabajo. \\
\hline Impresión de clientes & - Productos de calidad invariable. \\
& - Trato ecuánime y atención esmerada. \\
& - Precio justo. \\
\hline Interés por intervenir de los Accionistas. & - Adecuada retribución al capital invertido. \\
\hline VARIABLES INDEPENDIENTES & - Seguridad de la inversión. \\
\hline Expansión & - Buen nombre y reputación de la empresa. \\
\hline Rentabilidad de la inversión & INDICADORES \\
\hline
\end{tabular}


doce trabajadores. De allí se hizo una estratificación por distritos: se tomaron el $20 \%$ de empresas del Cercado de Lima; $10 \%$ de Lince; $10 \%$ de La Victoria; $10 \%$ de Jesús María, $20 \%$ de Ate; $20 \%$ de Miraflores y $10 \%$ de empresas de Surco.

- Los datos e informaciones de los balances de situación, el Estado de Pérdidas y Ganancias y los informes técnicos gerenciales fueron obtenidos del Ministerio de Trabajo y Promoción del Empleo.

La operatividad de las variables se realizó de la siguiente manera (ver Cuadro 2):

Cuadro 2

Indicadores de las Variables.

\begin{tabular}{|c|c|c|c|}
\hline$\longrightarrow$ afectan & $\longrightarrow$ & \multicolumn{2}{|c|}{$\begin{array}{l}\text { indicadores de las } \\
\text { Variables } \\
\text { Dependientes }\end{array}$} \\
\hline $\begin{array}{ll} & \text { Remuneraciones equitativa } \\
\text { - } & \text { Consideración personal en el trato } \\
\text { - } & \text { Buenas condiciones ambientales de trabajo } \\
\text { - } & \text { Productos de calidad invariable } \\
\text { - Trato ecuánime y atención esmerada } & \text { Precio justo }\end{array}$ & \begin{tabular}{|c|} 
volumen de \\
ventas
\end{tabular} & $x$ & $\begin{array}{c}\text { precio de venta } \\
\text { unitario }\end{array}$ \\
\hline $\begin{array}{l}\text { - Adecuada retribución al capital invertido } \\
\text { - Seguridad de la inversión } \\
\text { - Buen nombre y reputación de la empresa }\end{array}$ & \multicolumn{3}{|c|}{$\begin{array}{l}\text { - Utilidad neta después de impuesto } \\
\text { - Dividendo de las acciones } \\
\text { - Patrimonio neto } \\
\text { - Valor comercial y yominal de las acciones }\end{array}$} \\
\hline
\end{tabular}

Según esto, podemos ver que si hay un mejoramiento en los tres indicadores de la variable independiente éste afectará el incremento de los indicadores de las variables dependientes; considerando que si faltara alguna variable independiente no se produciría el aumento de la expansión, ni el incremento de la rentabilidad de inversión.

En los balances de situación y en los Estados de Pérdidas y Ganancias de las empresas analizadas se obtuvieron datos e informaciones de los últimos cinco años que se requerirán para obtener los volúmenes de venta y la rentabilidad de la inversión. Así mismo se obtuvieron los informes técnicos de los gerentes de personal, gerentes de marketing y gerentes financieros sobre los objetivos, políticas y estrategias, también de los últimos cinco años. Todo ello se complementó con encuestas para conocer cómo se manejaron los indicadores de las variables independientes. Luego se compararon los resultados de los informes técnicos gerenciales y los resultados de las encuestas con los resultados de los balances de situación y los resultados del Estado de Pérdidas y Ganancias. En esta forma procedimental se determinó el diseño de investigación.

\section{RESULTADOS}

Las estadísticas y la encuesta efectuada entre los empleados permitieron encontrar los siguientes resultados: en general, los trabajadores de las empresas analizadas mostraron una conducta regular; en cambio los trabajadores que laboran en el área de marketing tenían un correcto comportamiento y su aportación en el aumento de la expansión era de $60 \%$ y en la rentabilidad era de $65 \%$.

En los resultados estadísticos y encuesta aplicada sobre los clientes, se observó que la reacción de los clientes fue mejor que la de los empleados: cerca del 70\% de los clientes regulares compraron el $78 \%$ del volumen total de ventas; el resto de clientes mantuvieron una adquisición estable en las ventas, aunque todos estaban satisfechos con el trato, la calidad del producto y el precio.

En cambio los resultados de la estadística y encuestas sobre los accionistas no fue de lo mejor, ya que la reacción fue negativa. No estaban conformes con la retribución al capital invertido ni con la seguridad de la inversión, ni con la reputación de la empresa. Cerca del $80 \%$ de los accionistas no pensaban aumentar el capital social de la empresa y el $20 \%$ restante decía que podían aumentar el capital social siempre y cuando mejorara la rentabilidad de la inversión.

\section{DISCUSIÓN}

La apreciación es que las tres variables independientes -en su conjunto- no afectan a las variables dependientes; ello sólo se da en las variables independientes empleados y clientes que sí se afectan cuando hay una remuneración equitativa, un buen trato y buenas condiciones de trabajo, lo que redunda en un aumento en la expansión y en una mejor rentabilidad sobre la inversión. Lo mismo sucede con la variable independiente, ya que los clientes afectan el aumento de la expansión y una mejor rentabilidad.

Dentro de las variables independientes los accionistas se muestran indiferentes, no se conoce su reacción, y solo el $20 \%$ está listo para aumentar el capital social, ello a pesar que dos de las tres variables independientes (empleados y clientes), afectaron positivamente la expansión 
y rentabilidad; si los accionistas tuvieran interés en invertir se aumentaría el capital social que redundarían en mayor expansión y rentabilidad. $\mathrm{Si}$ se hubieran presentado las tres variables (empleado, cliente y accionistas) de manera conjunta, se hubiera mejorado notablemente la expansión y rentabilidad.

\section{CONCLUSIONES}

1. La expansión y la rentabilidad pueden ser mejoradas por la conducta de los empleados, la imagen que tienen los clientes de la empresa y por el interés de invertir de los accionistas.

2. Se debe de motivar en forma permanente la conducta de los empleados, satisfacer siempre a los clientes y estimular el interés por invertir por parte de los accionistas.

3. Se debe de incrementar el porcentaje de participación de los empleados, de los clientes y de los accionistas.

4. Se deben incrementar los indicadores de las variables empleados, clientes y accionistas.
5. Las variables independientes denominadas empleados, clientes y accionistas actúan como causas y como efectos de las variables dependientes denominadas expansión y rentabilidad.

\section{BIBLIOGRAFÍA}

Anthony, Robert N. (2003). Sistemas de Control de Gestión. Décima Edición. Madrid, Ed. Mc Graw-Hill.

Paredes, Alfredo Pezo. (2002). Gestión estratégica del capital humano. Lima, Programa Marco Profesional Tecnológico y Pedagógico en Perú (FORTE-PE).

Miro, Eliseo C. (1976). Estrategia Empresarial de Planificación de Objetivos. Buenos Aires - Argentina, Ed. Machi.

Van Horne, James C. (1979). Fundamentos de Administración Financiera. Madrid-España, Ed. Dossat.

Hernández Sampieri, M. en Roberto, C. (2003) Metodología de la Investigación. Tercera Edición. México, Ed. Mc. Graw-Hill. 